\title{
The diagnostic value of grey-scale inversion technique in chest radiography
}

\author{
Roberta Eufrasia Ledda ${ }^{1} \cdot$ Mario Silva $^{1} \cdot$ Nicole McMichael $^{2} \cdot$ Carlotta Sartorio $^{1} \cdot$ Cristina Branchi $^{1}$. \\ Gianluca Milanese $^{1} \cdot$ Sundeep M. Nayak ${ }^{3} \cdot$ Nicola Sverzellati $^{1}$
}

Received: 2 August 2021 / Accepted: 3 January 2022 / Published online: 18 January 2022

(c) The Author(s) 2022

\begin{abstract}
Purpose We investigated whether the additional use of grey-scale inversion technique improves the interpretation of eight chest abnormalities, in terms of diagnostic performance and interobserver variability.

Material and methods A total of 507 patients who underwent a chest computed tomography (CT) examination and a chest radiography (CXR) within $24 \mathrm{~h}$ were enrolled. CT was the standard of reference. Images were retrospectively reviewed for the presence of atelectasis, consolidation, interstitial abnormality, nodule, mass, pleural effusion, pneumothorax and rib fractures. Four CXR reading settings, involving 3 readers were organized: only standard; only inverted; standard followed by inverted; and inverted followed by standard. Sensitivity, specificity, positive predictive value (PPV), negative predictive value (NPV) and accuracy, assessed with the area under the curve (AUC), and their 95\% confidence interval were calculated for each reader and setting. Interobserver agreement was tested by Cohen's K test with quadratic weights $\left(K_{w}\right)$ and its $95 \% \mathrm{CI}$. Results CXR sensitivity \% for any finding was 35.1 (95\% CI: 33 to 37) for setting 1, 35.9 (95\% CI: 33 to 37), for setting 2, 32.59 (95\% CI: 30 to 34 ) for setting 3, and 35.56 (95\% CI: 33 to 37) for setting 4; specificity \% 93.78 (95\% CI: 91 to 95), 93.92 (95\% CI: 91 to 95), 94.43 (95\% CI: 92 to 96), 93.86 (95\% CI: 91 to 95); PPV \% 56.22 (95\% CI: 54.2 to 58.2), 56.49 (95\% CI: 54.5 to 58.5 ), 57.15 (95\% CI: 55 to 59), 56.75 (95\% CI: 54 to 58); NPV \% 85.66 (95\% CI: 83 to 87 ), 85.74 (95\% CI: 83 to 87 ), 85.29 (95\% CI: 83 to 87), 85.73 (95\% CI: 83 to 87 ); AUC values 0.64 (95\% CI: 0.62 to 0.66 ), 0.65 (95\% CI: 0.63 to 0.67 ), 0.64 (95\% CI: 0.62 to 0.66 ), 0.65 (95\% CI: 0.63 to 0.67$) ; K_{w}$ values 0.42 (95\% CI: 0.4 to 0.44 ), 0.40 (95\% CI: 0.38 to 0.42 ), 0.42 (95\% CI: 0.4 to 0.44 ), 0.41 (95\% CI: 0.39 to 0.43 ) for settings $1,2,3$ and 4 , respectively.
\end{abstract}

Conclusions No significant advantages were observed in the use of grey-scale inversion technique neither over standard display mode nor in combination at the detection of eight chest abnormalities.

Keywords Chest radiography $\cdot$ Grey-scale inversion $\cdot$ Diagnostic performance of chest radiography $\cdot$ Interobserver agreement

Roberta Eufrasia Ledda and Mario Silva have equally contributed to this work.

Gianluca Milanese

gianluca.milanese@unipr.it

1 Department of Medicine and Surgery, University of Parma, Scienze Radiologiche, University Hospital of Parma, Padiglione Barbieri, Via Gramsci 14, 43126 Parma, Italy

2 Department of Radiology Diagnostics, Skåne University Hospital of Malmö, Malmö, Sweden

3 Department of Radiology, Kaiser Permanente Northern California, San Leandro, CA, USA

\section{Abbreviations}

AP Anteroposterior

AUC Area under the curve

CT Computed tomography

CXR Chest X-ray

LL Left-lateral

NPV Negative predictive value

PA Posteroanterior

PACS Picture archiving and communication system

PPV Postive predictive value 


\section{Introduction}

Chest radiography (CXR) is generally considered entry level imaging to screen many pulmonary diseases with good performance as a screening uptake evaluation $[1,2]$. The interface between the bronchial tree, containing air, and structures with no air gives the radiographic image a natural contrast, used to advantage radiological interpreters (author radiologists) to depict abnormal findings [3]. These intrinsic anatomical features, along with continuous technical advancements in the field of digital radiography, have significantly contributed to make chest radiography one of the most requested radiological investigations [1, 4].

Over the last decades, digital chest radiography has iteratively and incrementally improved, with numerous processing tools being developed to support radiologists in the detection of pathological findings [2, 4, 5]. Most of these tools have been implemented to improve nodule detection, including digital tomosynthesis [6-8], dual energy and temporal subtraction techniques [9-11], computer-aided detection systems [12,13] and dark-field CXR. More recently, dark-field CXR has been demonstrated to be a valuable complementary tool for the assessment of pulmonary infiltrates, cardiomegaly and hemopericardium $[14,15]$. Such techniques are not yet widely available, and their use requires further validation. In comparison, the grey-scale inversion technique is universally available, being a built-in feature on most Picture Archiving and Communication System (PACS) display workstations. Based on the evidence that viewing the inverted image (black on white) improves human contrast perception [16], grey-scale inversion has been proposed as a valid supplementary tool to increase the diagnostic accuracy of radiographic imaging [17-21]. In chest radiography, the diagnostic value of inverted images has been investigated mostly for parenchymal nodules [17, 22-26], pneumothorax [20] and rib fractures [27] detection. The clinical advantages of using this display method, however, are still debated, and no general consensus has been reached.

The purpose of this study is to investigate whether the additional use of grey-scale inversion technique improves the interpretation of the main chest abnormalities, in terms of both diagnostic performance and interobserver variability.

\section{Material and methods}

\section{Ethics statement}

This study was approved by the Institutional Review Board of the University Hospital of Parma (Prot. 51059). Given the retrospective nature of the study, informed consent was waived.

\section{Study group}

The study selection criteria were as follows: chest CT examination and CXR obtained within $24 \mathrm{~h}$ of each other, in patients older than 18 years of age admitted to the University Hospital of Parma between October 2017 and October 2019. CTs and CXRs images affected by motion artefacts or other technical limitations (e.g. chest structures only partially included within the CT acquisition volume or the CXR projection) were excluded. Chest $\mathrm{CT}$ served as standard of reference (CT technique is reported in Supplementary material).

\section{CXR imaging technique}

Posteroanterior (PA) and left-lateral (LL) images were obtained with the patient standing up and in full inspiration with three digital radiography systems (Axiom Aristos FX, Siemens Healthineers; Essenta DR, Philips and DigitalDiagnost, Philips). Acquisition parameters were as follows: $125 \mathrm{kV}, 1.6 \mathrm{mAs}$, antiscatter grid with a $180 \mathrm{~cm}$ focus-detector distance.

Anteroposterior (AP) images were acquired with the patient either lying down or sitting up with two computed radiography systems (Practix 33 Plus, Philips and Practix 300, Philips). Acquisition parameters were as follows: $95-98 \mathrm{kV}, 3.2 \mathrm{mAs}$, with a $120 \mathrm{~cm}$ focus-detector distance.

Images were visualized on a dedicated workstation (BARCO visualization system, Kortrijk, Belgium), and greyscale inversion was performed through a built-in software of our PACS workstations (suite Estensa, Esaote, Genova, Italy) (Figs. 1 and 2).

\section{Data collection and interpretation}

$C X R$-Images of CXR were retrieved from the local PACS and independently reviewed by one general radiologist with 18 years of experience (Reader 1) and two third- year radiology residents (Readers 2 and 3), for the presence of eight predefined findings: atelectasis, consolidation, interstitial abnormality, nodule, mass, pleural effusion, pneumothorax and rib fractures. Chest abnormalities were classified based on the Fleischner Society glossary [28]. Standard grey-scale (also called "white bones") and inverted grey-scale ("black bones") CXRs were evaluated in two separate reading sessions, as follows:

- Session 1: standard setting first, followed by inverted grey-scale

- Session 2: inverted grey-scale first, followed by standard. 
Fig. 1 Representative example of right apical pneumothorax (arrows) in standard (A) and inverted grey-scale (B) CXR images (posteroanterior projection)
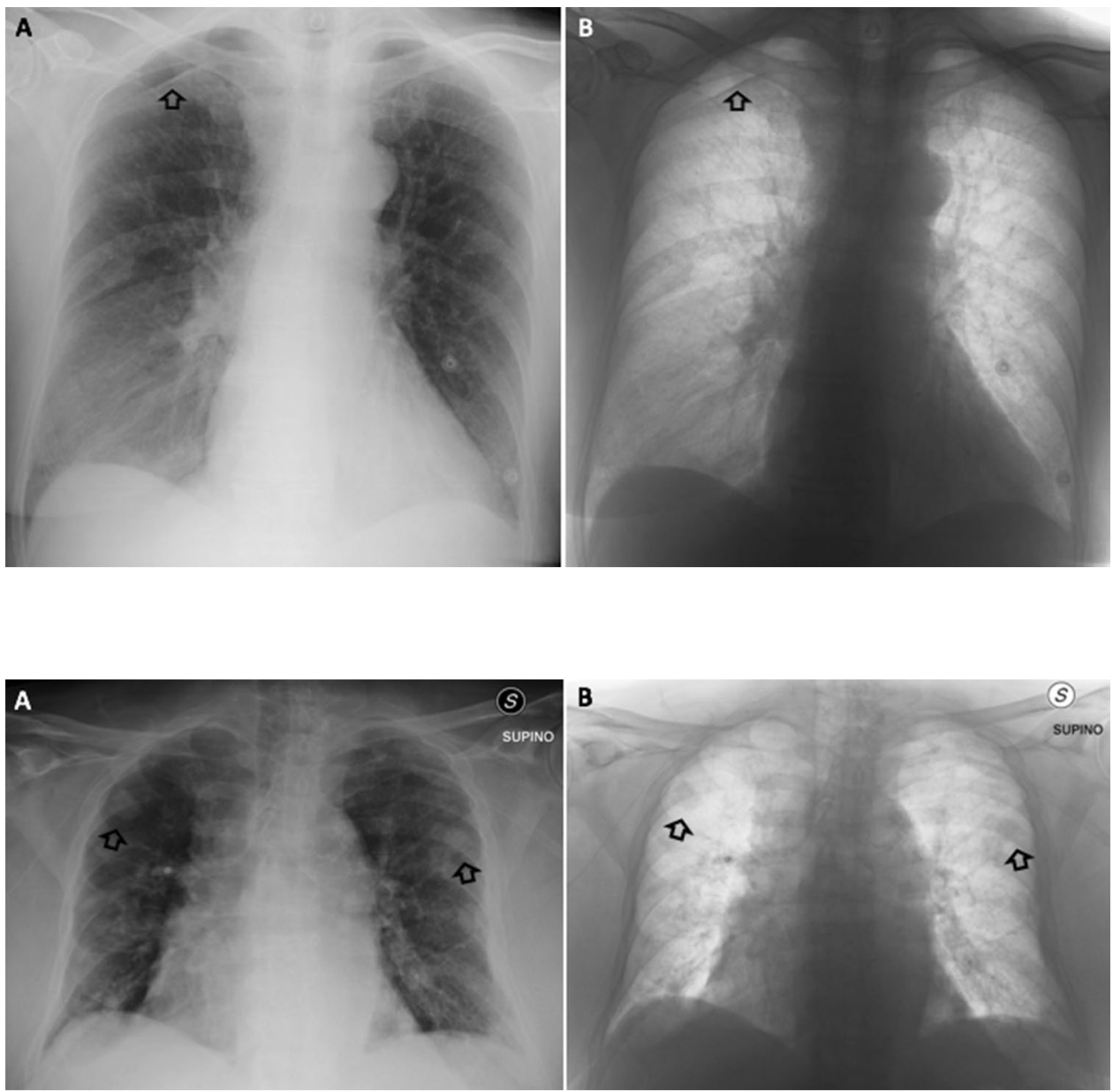

Fig. 2 Representative example of bilateral parenchymal nodules (arrows) in standard (A) and inverted grey-scale (B) CXR images (anteroposterior projection)
Any discrepancy between Readers 4 and 5 was resolved by a chest radiologist with 13 years of experience.

The same classification system was applied to discretize CXR outcome in binary categories.

findings was recorded separately for standard and inverted grey-scale to analyse the findings by either first line standard or inverted. Subsequently, the adjunct findings by consecutive reading with either approach were recorded. This database allowed testing of CXR accuracy and interobserver agreement under different reading settings and combinations (see Statistical analysis). Reading time was recorded for each reader and session.

Standard of reference-The diagnostic performance of CXR with different visualization modes was tested against CT, as standard of reference. CT images were reviewed independently by two resident radiologists (Readers 4 and 5 , respectively) who had access to the radiological reports, and classified into positive or negative, as follows:

- Positive CT was assigned in case of at least one of the eight above-mentioned findings;

- Negative CT was assigned when none of them was present.

\section{Statistical analysis}

Continuous data were expressed as median and its $95 \%$ confidence interval (95\% CI), whereas categorical data were expressed as absolute and relative distribution, with corresponding 95\% CI using Wilson method.

The following reading settings were assembled for comparison with CT standard of reference:

- Setting 1: standard reading only, derived from session 1

- Setting 2: inverted reading only, derived from session 2

- Setting 3: combined reading, first standard followed by inverted reading as per full session 1

- Setting 4: combined reading, first inverted followed by standard reading as per full session 2 
Sensitivity, specificity, positive predictive value (PPV) and negative predictive value (NPV) were calculated for each reader and all reading settings; accuracy was tested with the area under the curve (AUC) values and its 95\% confidence interval $(95 \% \mathrm{CI})$. Interobserver agreement was tested by Cohen's K test with quadratic weights $\left(k_{w}\right)$ and its 95\%CI: $k_{w}<0.20$ was considered to indicate poor agreement, $0.21<k_{w}<0.40$ fair agreement, $0.41<k_{w}<0.60$ moderate agreement, $0.61<k_{w}<0.80$ good agreement, and $0.81<k_{w}<1.00$ very good agreement.

A $p$ value $<0.05$ was deemed statistically significant. Statistical analysis was performed by MedCalc Software bvba (version 19.1-64-bit, Ostend, Belgium).

\section{Results}

\section{Study population}

A total of 553 consecutive patients underwent a chest CT and CXR within $24 \mathrm{~h}$ of each other, at the University Hospital of Parma between October 2017 and October 2019. Forty-six $(8.32 \%, 95 \%$ CI $6.3 \%$ to $10.92 \%)$ patients were excluded because of CT and/or CXR technical limitations: $31 / 46(67.39 \%, 95 \%$ CI $52.97 \%$ to $79.13 \%)$ because of CT motion artifacts; $15 / 46(32.61 \%, 95 \%$ CI $20.87 \%$ to $47.03 \%)$ due to chest structures only partially included within the CT acquisition volume or CXR projections. A total of 507 (median age $69.95 \%$ CI 66.94 to $71 ; 285 / 507$ men, 56.2\%) patients were enrolled (Fig. 3). Main clinical indications for both CT and CXR included trauma, chest pain, dyspnea, fever and persistent cough.

\section{CT findings}

A total of $393 / 507(77.5 \%, 95 \%$ CI $73.68 \%$ to $80.93 \%)$ CTs were scored positives and $114 / 507(22.5 \%, 95 \% \mathrm{CI} 19.07 \%$ to $26.32 \%$ ) negatives. Detailed distribution of CT pathological findings is reported in Table 1, whereas CT acquisition data in Supplementary material.

\section{CXR acquisition data}

PA and LL projections were performed in 254/507 (50.1\%, 95\% CI $45.76 \%$ to $54.44 \%$ ), whereas AP projection was performed in $253 / 507(49.9 \%, 95 \%$ CI $45.56 \%$ to $54.24 \%)$. The
Fig. 3 Flow chart of patient selection

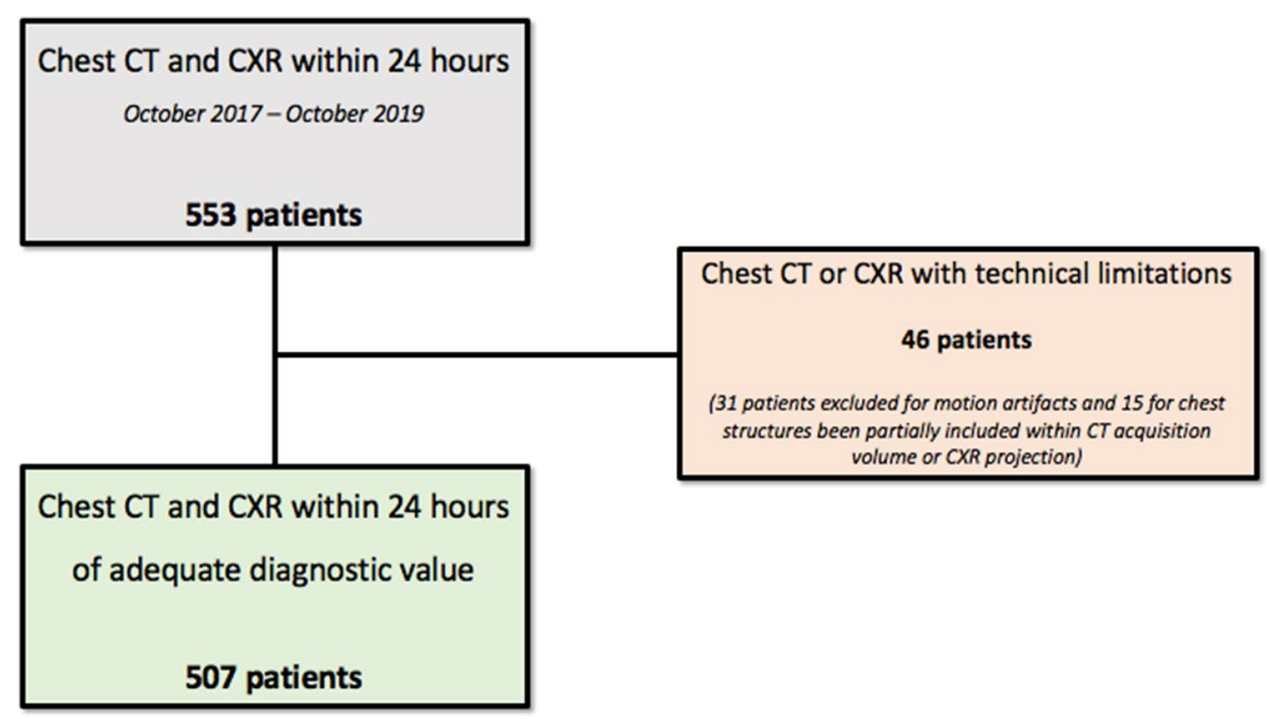

Table 1 Distribution of chest CT pathological findings

\begin{tabular}{lll}
\hline Findings & $\mathrm{N}^{\circ}$ & $\%$ [95\%CI] \\
\hline Atelectasis & $166 / 507$ & $32.7[28.8-36.94]$ \\
Consolidation & $175 / 507$ & 34 [30.51-38.76] \\
Interstitial abnormalities & $67 / 507$ & $13.2[10.54-16.44]$ \\
Nodule (median size 7 mm, 95\%CI 6-8) & $85 / 507$ & $16.8[13.77-20.27]$ \\
Mass (median size 55 mm, 95\%CI 38.5-73.75) & $10 / 507$ & $1.97[1.07-3.59]$ \\
Pleural effusion & $167 / 507$ & 32.9 [28.99-37.15] \\
Pneumothorax (median size 23 mm, 95\%CI 9.75-35.25) & $28 / 507$ & $5.5[3.85-7.86]$ \\
Rib fractures & $65 / 507$ & $12.8[10.19-16.01]$ \\
\hline
\end{tabular}


effect of reading setting was comparable for both standing and supine CXR imaging.

\section{Reading time}

The median reading time of session 1 was 79 s [ $95 \%$ CI, 77 to $85 \mathrm{~s}$ ] for Reader 1, 84 [95\% CI, 80 to $88 \mathrm{~s}$ ] for Reader 2, and 83 [95\% CI, 80.5 to 87 s] for Reader 3, whereas that of session 2 was $61 \mathrm{~s}$ [95\%CI, 57 to $64 \mathrm{~s}$ ] for Reader 1 and 59 for Readers 2 [95\%CI, 57 to 60.7 s] and 3 [95\%CI, 55 to 60 s].

\section{Diagnostic performance of CXR}

Overall, sensitivity of CXR for any finding ranged 7.1-60\% for setting 1, and $8.2-60 \%$ for each of setting 2,3 , and 4 . Specificity ranged $76.5-99.8 \%, 78-99.8 \%, 73.3-100 \%$, $73-100 \%$, for settings $1,2,3$ and 4 , respectively. PPV ranged $16.7-90 \%, 17.2-90.9 \%, 20-100 \%, 17.8-100 \%$, for settings 1, 2, 3 and 4, respectively. NPV ranged 69.6-99.2\%, $69.3-99.2 \%, 69.5-99.2 \%, 69.8-99.2 \%$, for settings $1,2,3$ and 4, respectively. AUC ranged $0.529-0.781,0.527-0.779$, $0.531-0.779,0.529-0.779$, for settings $1,2,3$ and 4 , respectively. Overall, CXR accuracy was not significantly improved by the inverted images compared to setting 1 . For Reader 3, CXR sensitivity was improved by the combined reading at the detection of consolidation in setting 4 and of pneumothorax and rib fractures in setting 3 , whereas for Reader 1 the combined approach improved CXR PPV at the detection of pleural effusion in setting 3. Sensitivity, specificity, PPV, NPV and AUC values are detailed for each radiological finding in Table 2.

\section{Interobserver agreement}

$K_{w}$ values for any finding ranged $0.23-0.63,0.13-0.73$, $0.21-0.66,0.14-0.75$ for settings $1,2,3$ and 4 , respectively. Regardless of size, interobserver agreement at the detection of pneumothorax between the residents and the senior radiologist showed a slight improvement in both settings 3 and 4 (Table 3 ). $K_{w}$ values were generally higher for large pneumothorax-sized $\geq 3 \mathrm{~cm} \mathrm{[29]—with} \mathrm{only} \mathrm{two} \mathrm{excep-}$ tions of greater values observed for small pneumothoraces (sized $<3 \mathrm{~cm}$ ). Details are reported in Table 4.

\section{Discussion}

We observed that grey-scale inversion display mode did not significantly improve diagnostic performance or interobserver agreement compared with standard viewing mode. Combinations of standard and inverted modes could help in reducing the interobserver variability across different levels of expertise.
The visualization of CXR is usually performed by "white bones" mode on video-terminal; however, the perception of CXR images is also (variably) preferred with "black bones" mode. The latter represents a subjective adaptation of the standard setting, based on the individual feeling that the detection of abnormal findings is eased by the inverted images. We undertook this study for systematic evaluation of such perception and showed that there is no actual diagnostic difference. Our results partially confirm previous observation from Park et al. who investigated sensitivity and accuracy of the grey-scale inversion technique, limited to the detection of rib fractures. Park reported that the combination of the two reading modalities could improve chest radiography sensitivity and accuracy among residents and medical students, namely among readers with limited experience [27]. In our study, the combined use of the two approaches increased CXR sensitivity at the detection of consolidation for one resident, when using reading setting 4 (i.e. first inverted, followed by standard), pneumothorax and rib fractures in setting 3 (i.e. first standard, followed by inverted) and 4. However, the improvement did not reach statistical significance for accuracy performance.

Interobserver agreement at the detection of pneumothorax between the residents and the senior radiologist showed a moderate improvement in both sessions and, as expected, was generally higher for large pneumothoraces in all settings and among all readers. Since the required reading time for both sessions was relatively short (not greater than $84 \mathrm{~s}$ ), the combined use of the two display modes might be worth exploiting when pneumothorax is suspected. Having said that, pneumothorax was scarcely represented among the enrolled patients (5.5\%, 28 cases).

The combined reading approach improved the PPV at the detection of pleural effusion by the senior radiologist, but showed a general drop in diagnostic performance as compared to the standard approach for the same reader. The unfamiliarity with the "black bones" images might have affected their interpretation by the senior radiologist. As pointed out by McMahon et al., when a new type of image results in lesser accuracy, the unfamiliarity with the new approach must be taken into account prior to blaming intrinsic properties of the new modality [11]. This "unfamiliarity effect" tends to have a minor impact on younger author radiologists, who are inevitably less affected by a long-lasting habit.

Thompson et al. reported that two display modes can improve nodule detection [26]. These authors hypothesized that the advantage of using two display modes might lie in the fast-flicking between the two images, namely standard and inverted, which would draw attention to suspicious areas, (e.g. lung periphery). This fast-flicking technique was not employed by our readers, for whom the detection was already slightly improved, suggesting that it might only 


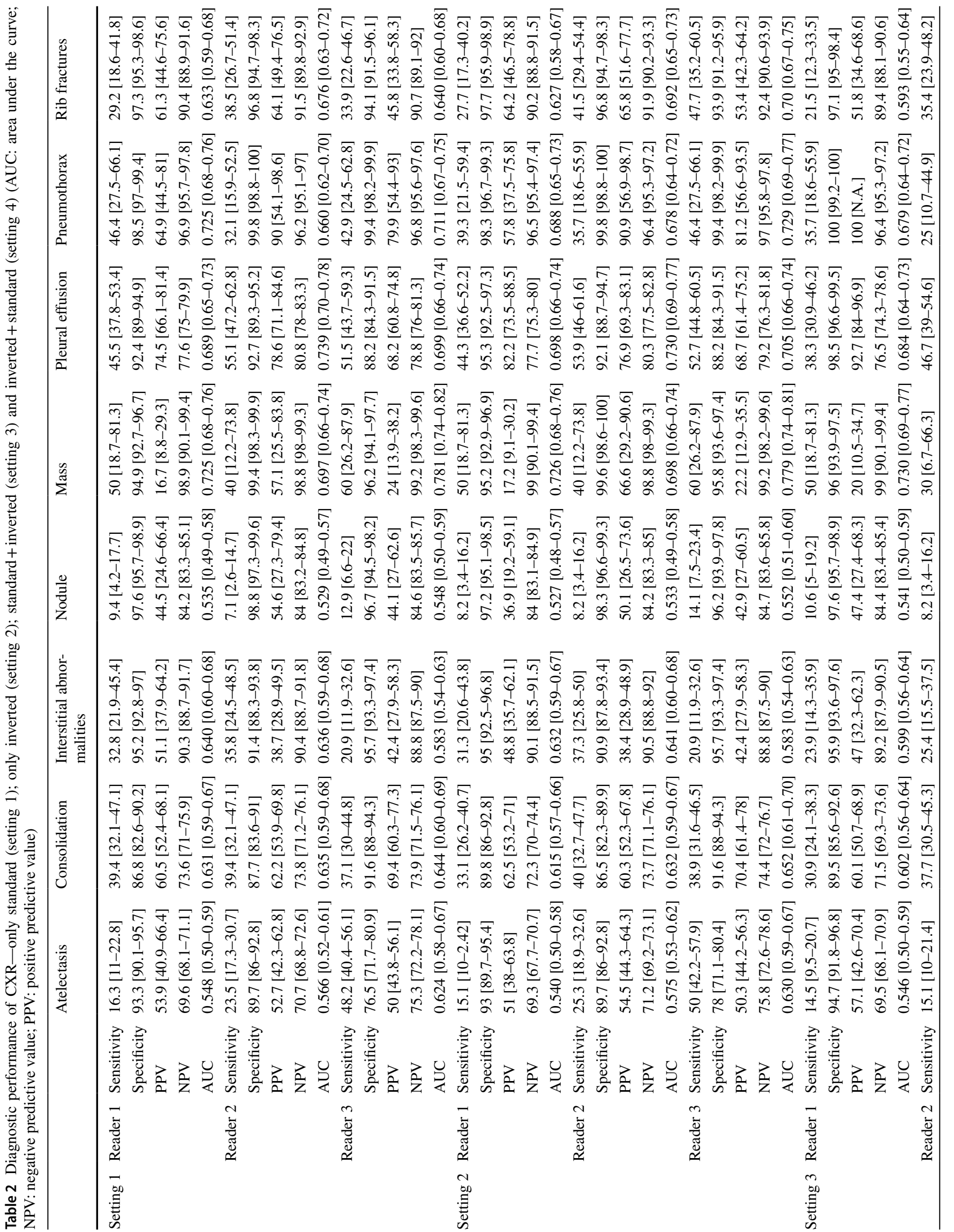




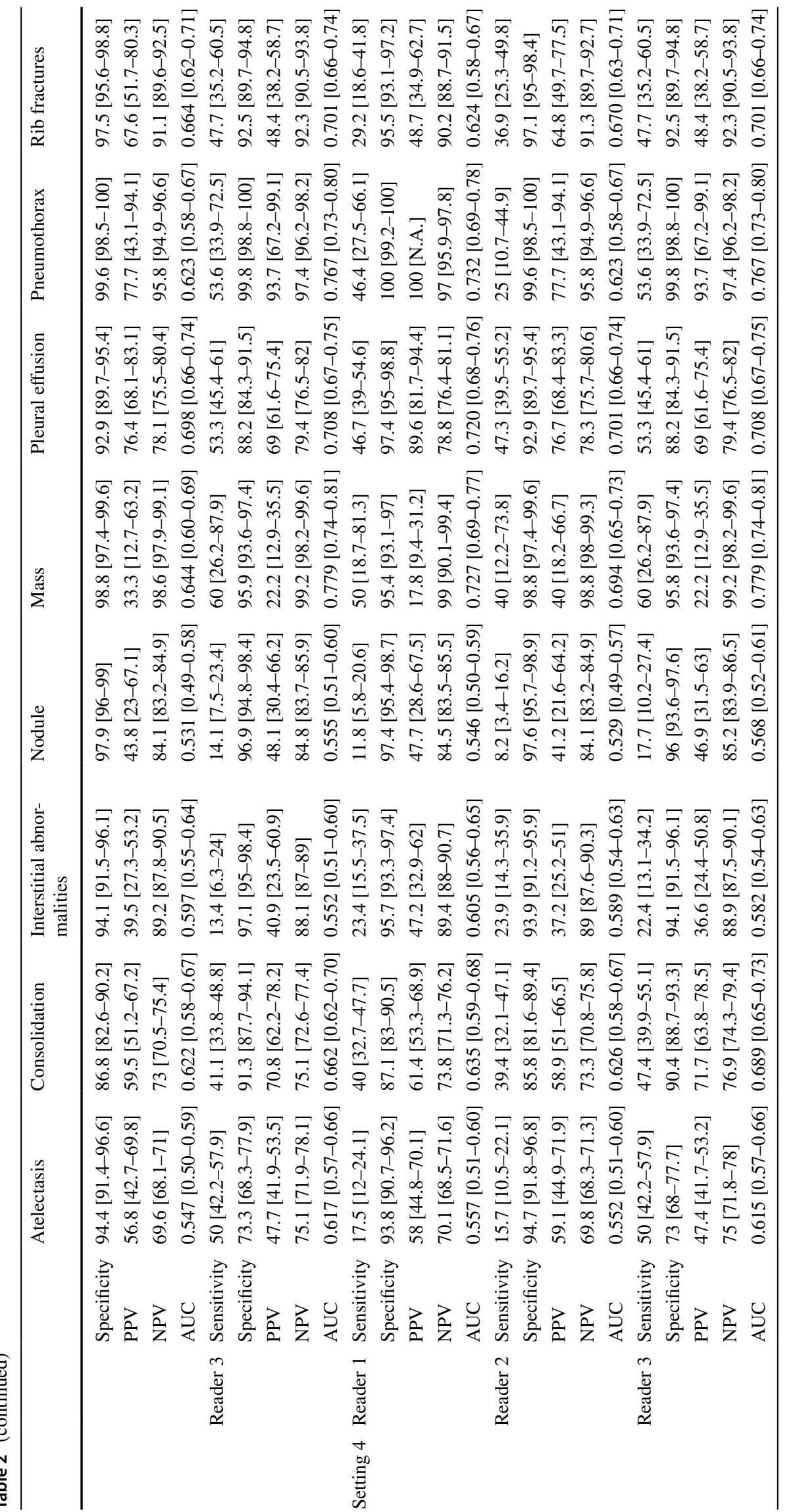




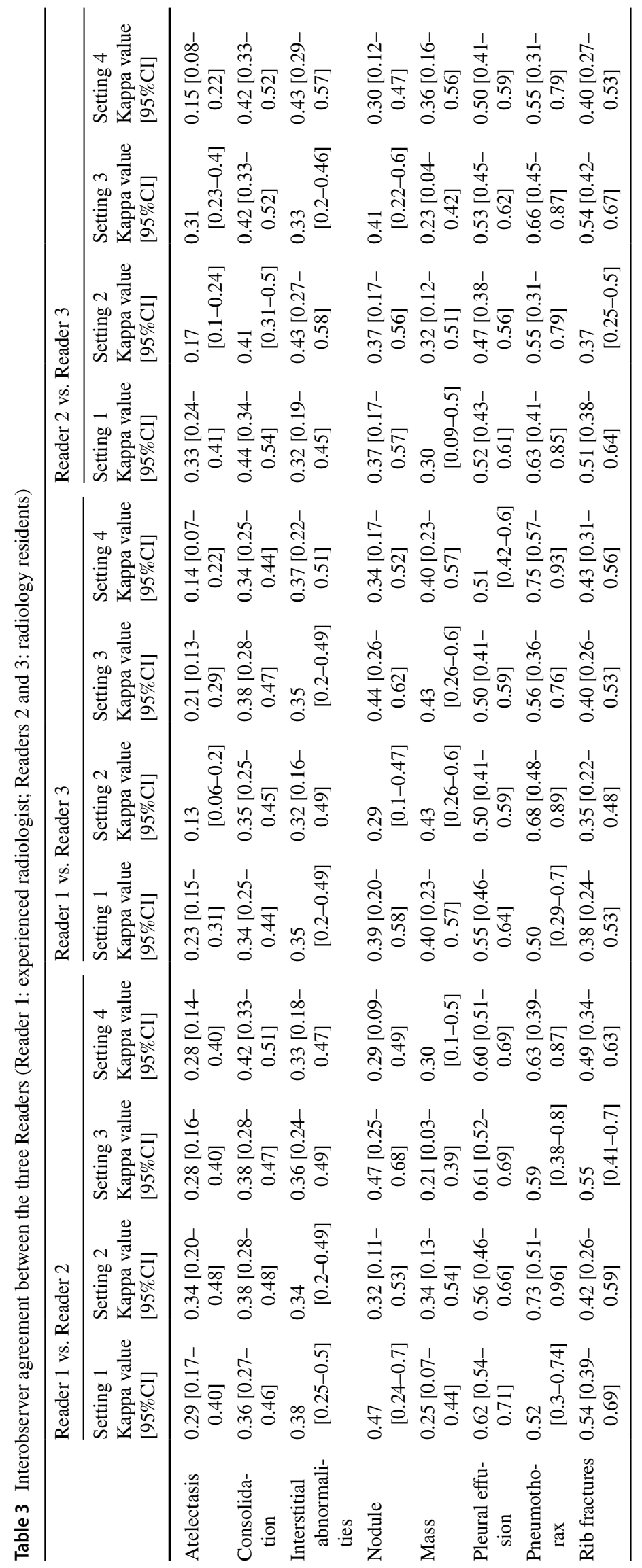




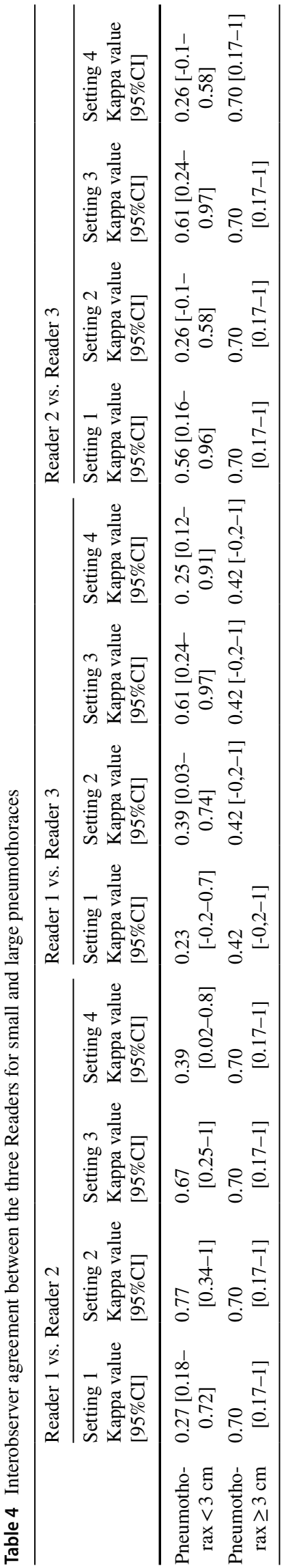

partly explain the advantages of such a combination. Even if limited in number, the majority of studies that have applied the grey-scale inversion display mode to chest radiography have attempted to demonstrate its additional value in detecting lung nodules, either real or simulated, with opposite results $[17,22-26]$. Nodules were fairly represented in our sample (16.8\%, 85 cases), and significant differences were not observed in accuracy or interobserver agreement with the combination of the two techniques. Their depiction rate was generally low among the three readers, ranging $7.1 \%$ to $17.7 \%$. One of the reasons of such low percentages can be found in their relatively small size (nodule median diameter of $7 \mathrm{~mm}, 95 \% \mathrm{CI}, 6$ to $8 \mathrm{~mm}$ ), which has likely contributed to reduce their detectability by CXR. Previous studies reported better performance in nodule detection, notably with relatively larger solid nodules [17]. As opposite to previous analyses, a nodule size range was not set at the time of patient selection (22), since the general intent of this investigation was to reproduce a real clinical setting, without focusing on a pre-defined finding.

To our knowledge, this is the first study testing eight different abnormal findings at the same time and within such a large population. Indeed, the majority of studies that have investigated the application of grey-scale inversion display mode into chest radiography only tested one selected finding at time, enrolling no more than 300 subjects. Furthermore, we included bedside CXRs, with the aim of reproducing a real clinical setting, where a good proportion of patients is unable to stand (e.g. trauma patients or severely ill ones). Of note, the effect of reading setting was comparable for both standing and supine CXR imaging.

Our study, however, has several limitations. First, the retrospective design is prone to confounding factors, such as selection of patients. Second, CXRs were obtained with different technical equipment and parameters, which can ultimately affect the detectability of findings, nonetheless representing the actual routine of this imaging modality. Third, some of the findings included in the analysis were barely represented within the sample, such as mass $(1.97 \%$, 10 cases). Finally, the presence of only one senior radiologist limited the possibility of investigating the impact of different levels of expertise.

In conclusion, we observed no significant advantages in the use of grey-scale inversion technique in expert radiologist. The combination of grey-scale inversion display modes with standard mode could reduce the interobserver variability in readers with limited expertise.

Supplementary Information The online version contains supplementary material available at https://doi.org/10.1007/s11547-022-01453-0.

Authors' contributions MS, SN and NS were involved in the conception and design. NM, CS and CB contributed to the provision of study materials. NM, CS, CB and REL contributed to the collection and 
assembly of data. REL and MS were involved in the data analysis and interpretation. REL, MS, SN and GM contributed to the manuscript writing. All authors contributed to the final approval of manuscript.

Funding This research did not receive any specific grant from funding agencies in the public, commercial or not-for profit sectors.

Availability of data and material All data generated or analysed during this study are included in this published article and its supplementary information file.

\section{Declarations}

Conflict of interest The authors declare that they have no conflict of interest.

Ethical approval This study was approved by the Institutional Review Board of the University Hospital of Parma (Prot. 51059).

Consent to participate Given the retrospective nature of the study, informed consent was waived.

Consent for publication Not applicable.

Open Access This article is licensed under a Creative Commons Attribution 4.0 International License, which permits use, sharing, adaptation, distribution and reproduction in any medium or format, as long as you give appropriate credit to the original author(s) and the source, provide a link to the Creative Commons licence, and indicate if changes were made. The images or other third party material in this article are included in the article's Creative Commons licence, unless indicated otherwise in a credit line to the material. If material is not included in the article's Creative Commons licence and your intended use is not permitted by statutory regulation or exceeds the permitted use, you will need to obtain permission directly from the copyright holder. To view a copy of this licence, visit http://creativecommons.org/licenses/by/4.0/.

\section{References}

1. Levin DC, Parker L, Rao VM (2017) Recent trends in imaging use in hospital settings: implications for future planning. J Am Coll Radiol 14(3):331-336

2. McAdams HP, Samei E, Dobbins J 3rd, Tourassi GD, Ravin CE (2006) Recent advances in chest radiography. Radiology 241(3):663-683

3. Gibbs JM, Chandrasekhar CA, Ferguson EC, Oldham SA (2007) Lines and stripes: where did they go?-from conventional radiography to CT. Radiographics 27(1):33-48

4. Schaefer-Prokop C, Neitzel U, Venema HW, Uffmann M, Prokop M (2008) Digital chest radiography: an update on modern technology, dose containment and control of image quality. Eur Radiol 18(9):1818-1830

5. Tanaka R (2016) Dynamic chest radiography: flat-panel detector (FPD) based functional X-ray imaging. Radiol Phys Technol 9(2):139-153

6. Chou SH, Kicska GA, Pipavath SN, Reddy GP (2014) Digital tomosynthesis of the chest: current and emerging applications. Radiographics 34(2):359-372
7. Nagatani Y, Takahashi M, Ikeda M, Nitta N, Miyata K, Hanaoka $\mathrm{J}$ et al (2018) Sub-solid nodule detectability in seven observers of seventy-nine clinical cases: comparison between ultra-lowdose chest digital tomosynthesis with iterative reconstruction and chest radiography by receiver-operating characteristics analysis. Eur J Radiol 107:166-174

8. Petersson C, Bath M, Vikgren J, Johnsson AA (2016) An analysis of the potential role of chest tomosynthesis in optimising imaging resources in thoracic radiology. Radiat Prot Dosimetry 169(1-4):165-170

9. Kumar SG, Garg MK, Khandelwal N, Gupta P, Gupta D, Aggarwal AN et al (2015) Role of digital tomosynthesis and dual energy subtraction digital radiography in detecting pulmonary nodules. Eur J Radiol 84(7):1383-1391

10. Kuhlman JE, Collins J, Brooks GN, Yandow DR, Broderick LS (2006) Dual-energy subtraction chest radiography: what to look for beyond calcified nodules. Radiographics 26(1):79-92

11. MacMahon H, Li F, Engelmann R, Roberts R, Armato S (2008) Dual energy subtraction and temporal subtraction chest radiography. J Thorac Imaging 23(2):77-85

12. van Ginneken B, Hogeweg L, Prokop M (2009) Computer-aided diagnosis in chest radiography: beyond nodules. Eur J Radiol 72(2):226-230

13. De Boo DW, van Hoorn F, van Schuppen J, Schijf L, Scheerder MJ, Freling NJ et al (2012) Observer training for computeraided detection of pulmonary nodules in chest radiography. Eur Radiol 22(8):1659-1664

14. Fingerle AA, De Marco F, Andrejewski J, Willer K, Gromann LB, Noichl W et al (2019) Imaging features in post-mortem $\mathrm{X}$-ray dark-field chest radiographs and correlation with conventional X-ray and CT. Eur Radiol Exp 3(1):25

15. Hatabu H, Madore B (2021) Dark-field chest X-ray imaging: an evolving technique in the century-old history of chest X-ray imaging. Radiology 301(2):396-397

16. Blackwell HR (1946) Contrast thresholds of the human eye. J Opt Soc Am 36(11):624-643

17. De Boo DW, Uffmann M, Bipat S, Boorsma EF, Scheerder MJ, Weber M et al (2011) Gray-scale reversal for the detection of pulmonary nodules on a PACS workstation. AJR Am J Roentgenol 197(5):1096-1100

18. Kehler M, Albrechtsson U, Andresdottir A, Hochbergs P, Larusdottir H, Lundin A et al (1991) Efficacy of inverted digital luminescence radiography in evaluating chest neoplasms. Acta Radiol 32(6):442-448

19. MacMahon H, Metz CE, Doi K, Kim T, Giger ML, Chan HP (1988) Digital chest radiography: effect on diagnostic accuracy of hard copy, conventional video, and reversed gray scale video display formats. Radiology 168(3):669-673

20. Musalar E, Ekinci S, Unek O, Ars E, Eren HS, Gurses B et al (2017) Conventional vs invert-grayscale X-ray for diagnosis of pneumothorax in the emergency setting. Am J Emerg Med 35(9):1217-1221

21. Sakakura CE, Loffredo Lde C, Scaf G (2004) Diagnostic agreement of conventional and inverted scanned panoramic radiographs in the detection of the mandibular canal and the mental foramen. J Oral Implantol 30(1):2-6

22. Kirchner J, Gadek D, Goltz JP, Doroch-Gadek A, Stuckradt S, Liermann D et al (2013) Standard versus inverted digital luminescence radiography in detecting pulmonary nodules: a ROC analysis. Eur J Radiol 82(10):1799-1803

23. Lungren MP, Samei E, Barnhart H, McAdams HP, Leder RA, Christensen JD et al (2012) Gray-scale inversion radiographic 
display for the detection of pulmonary nodules on chest radiographs. Clin Imaging 36(5):515-521

24. Robinson JW, Ryan JT, McEntee MF, Lewis SJ, Evanoff MG, Rainford LA et al (2013) Grey-scale inversion improves detection of lung nodules. Br J Radiol 86(1021):27961545

25. Sheline ME, Brikman I, Epstein DM, Mezrich JL, Kundel HL, Arenson RL (1989) The diagnosis of pulmonary nodules: comparison between standard and inverse digitized images and conventional chest radiographs. AJR Am J Roentgenol 152(2):261-263

26. Thompson JD, Thomas NB, Manning DJ, Hogg P (2016) The impact of greyscale inversion for nodule detection in an anthropomorphic chest phantom: a free-response observer study. Br J Radiol 89(1064):20160249

27. Park JB, Cho YS, Choi HJ (2015) Diagnostic accuracy of the inverted grayscale rib series for detection of rib fracture in minor chest trauma. Am J Emerg Med 33(4):548-552
28. Hansell DM, Bankier AA, MacMahon H, McLoud TC, Muller NL, Remy J (2008) Fleischner Society: glossary of terms for thoracic imaging. Radiology 246(3):697-722

29. Baumann MH, Strange C, Heffner JE, Light R, Kirby TJ, Klein $\mathrm{J}$ et al (2001) Management of spontaneous pneumothorax: an American College of Chest Physicians Delphi consensus statement. Chest 119(2):590-602

Publisher's Note Springer Nature remains neutral with regard to jurisdictional claims in published maps and institutional affiliations. 